\title{
Lower total blood loss in total knee arthroplasty with a low-pressure tourniquet than without
}

\author{
Simon Joufflineau, Emmanuel ThienPont
}

From the Department of Orthopaedic Surgery, University Hospital Saint Luc-UCL, Brussels, Belgium

Tourniquet-use has been described in literature as a surgical factor leading to increased post-operative hidden blood loss in total knee arthroplasty (TKA). The hypothesis for this retrospective study was that low-pressure tourniquet TKA would offer the benefits of tourniquet surgery without the potential negative effects on late blood loss after the procedure. Therefore, this study evaluated total (TBL) and hidden blood loss (HBL) in TKA with or without a tourniquet. We retrospectively compared two groups: one group $(n=54)$ undergoing TKA without a tourniquet and one group with a low-pressure tourniquet $(n=110)$. The outcomes compared were hemoglobin levels 2 weeks before surgery and at days 2 and 4 after surgery to calculate total and hidden blood loss, transfusion rate, and functional outcome. A higher total blood loss was observed in the no tourniquet group with a mean loss of $1073 \mathrm{~mL}$ against $890 \mathrm{~mL}$ in the tourniquet group (p-value $=\mathbf{0 , 0 0 3})$. Hidden blood loss values between both groups were statistically not significant : in the no-tourniquet group, Hb drop between $\mathrm{D} 2$ and $\mathrm{D} 4$ was $0.5 \mathrm{~g} / \mathrm{dL}$ against $0.4 \mathrm{~g} /$ $\mathrm{dL}$ in the low-pressure tourniquet group. The tourniquet-less group did not present with better

Funding : No funding received.

Authorship contributions: Sj and Et designed the study protocol. Sj collected the data, searched the literature and performed the statistical analysis. Et and sj both wrote the paper.

Et gave final approval of the manuscript.

The authors declare no conflicts of interest with the present study. functional scores. This study showed that lowpressure tourniquet use reduces TBL without increasing $\mathrm{HBL}$ in TKA.

Keywords : blood loss ; total knee arthroplasty ; tourniquet ; hemoglobin.

\section{INTRODUCTION}

Total knee arthroplasty (TKA) is recognized as the preferred surgical treatment for advanced tricompartmental knee osteoarthritis (OA).

The advocates of fast track surgery claim early and less painful recovery without tourniquet use. Believers of tourniquet-less knee arthroplasty have mainly reported less pain, quicker recovery and better quadriceps function. They also pretend that tourniquets lead to more hidden blood loss (HBL) (1). Tourniquet-using surgeons are claiming a clearer operative field, a reduced surgical time, a dryer bone-implant interface during cementation and less overall blood loss (1-4).

Simon Joufflineau,

- Emmanuel Thienpont

Department of Orthopaedic Surgery, University Hospital Saint Luc-UCL, Brussels, Belgium

Correspondence : Emmanuel Thienpont, Department of Orthopaedic Surgery, Cliniques Universitaires Saint Luc, Av. Hippocrate 10, 1200 Brussels, Belgium.

Email : emmanuel.thienpont@uclouvain.be

- 2021, Acta Orthopædica Belgica.

Acta Orthopædica Belgica, Vol. 87 - 3 - 2021 
HBL is partly due to a combination of hemarthrosis, blood dilution, hemolysis and diffusion of blood into the soft tissues $(2,3)$.

Several authors tried to determine and calculate HBL. With its physiopathology remaining unclear, the methodology leading to HBL calculation varies from one study to another (4-6). Nevertheless, all surgeons are looking to decrease total blood loss (TBL) to reduce post-operative anemia and anemiaassociated co-morbidities such as blood transfusion, ischemia-related complications, infection and slower rehabilitation (7).

For several years, the senior author (ET) has used a low-pressure tourniquet. This could potentially combine the advantages of early recovery as for tourniquet-less arthroplasty, while keeping the advantages of tourniquet use. The purpose of this retrospective study was to evaluate the amount of total and hidden blood loss by hemoglobin drop and to evaluate early functional outcome in primary TKA with low-pressure tourniquet use.

\section{MATERIALS AND METHOD}

All demographic and medical data have been collected from the medical software used in our institution (Medical Explorer). The study (2019/23SEP/414) was approved by the ethics committee of the hospital. A cohort of 164 patients between November 2013 and March 2020 who underwent primary TKA for tricompartmental knee osteoarthritis was retrospectively studied. Demographic data are given in Table I.

In study group one, 54 patients have been operated without tourniquet. The first 26 because of a preoperative decision not to use it : 11 for arterial disease or previous arterial by-pass surgery, 7 for neuropathy, 2 for large thigh circumference, 2 for previous scars or skin fragility, 1 for myasthenia, 1 for thigh hemangioma, 1 for lymphoedema and 1 for high tobacco use. For the other 28 patients, tourniquet was released because of a venoustourniquet, within the first few minutes of the surgical approach.

In the second group, 110 patients were operated with a low-pressure tourniquet.

For both groups, criteria of inclusion were primary tricompartmental osteoarthritis. There were no age or BMI limitations for these adult patients.

The exclusion criteria were systemic diseases affecting blood hemoglobin level or the erythropoietic potential of the patients, large deformities of the leg or previous surgery that would increase the procedure time.

The average duration of the procedures was 87 minutes from incision to closure. Any perioperative event which would have prolonged the operating time beyond 95 minutes was considered as exclusion criterion.

Oral anticoagulation medicine was pre-operatively substituted by therapeutic low-molecularweight-heparin (LMWH) doses as recommended by anesthetics.

Table I. - Demographic data .

\begin{tabular}{|c|c|c|c|c|c|c|}
\hline \multicolumn{7}{|c|}{ No tourniquet } \\
\hline & \multicolumn{2}{|c|}{ Total $(n=54)$} & \multicolumn{2}{|c|}{ Men (n=18) } & \multicolumn{2}{|c|}{ Women $(n=36)$} \\
\hline & mean $(S D)$ & median & mean (SD) & median & mean (SD) & median \\
\hline Age (years) & $70,5(10)$ & 72 & $70(11)$ & 72,5 & $70,5(9,5)$ & 71,5 \\
\hline Weight (kg) & $85,7(21,1)$ & 87,0 & $87,0(14,6)$ & 89,5 & $85,0(23,9)$ & 84,0 \\
\hline Height (cm) & $167(10)$ & 165 & $175(11))$ & 176 & $164(7)$ & 163 \\
\hline BMI $\left(\mathrm{kg} / \mathrm{m}^{2}\right)$ & $30,7(7,7)$ & 28,7 & $28,6(5,5)$ & 27,5 & $31,7(8,5)$ & 30 \\
\hline \multicolumn{7}{|c|}{ Tourniquet } \\
\hline & \multicolumn{2}{|c|}{ Total $(n=110)$} & \multicolumn{2}{|c|}{ Men $(n=64)$} & \multicolumn{2}{|c|}{ Women $(n=46)$} \\
\hline & mean (SD) & median & mean (SD) & median & mean (SD) & median \\
\hline Age (years) & $67,5(9,5)$ & 69 & $67,5(10)$ & 68 & $68(9,5)$ & 69 \\
\hline Weight (kg) & $89,1(15,5)$ & 90,0 & $94,22(13,4)$ & 96,0 & $82,0(15,6)$ & 81,0 \\
\hline Height $(\mathrm{cm})$ & $171(10)$ & 171 & $177(7)$ & 176 & $162(7)$ & 164 \\
\hline BMI $\left(\mathrm{kg} / \mathrm{m}^{2}\right)$ & $30,5(4,8)$ & 29,6 & $30,0(3,7)$ & 29,5 & $31,2(6,0))$ & 30,1 \\
\hline
\end{tabular}


Daily intake of aspirin was continued until the day before surgery, as this does not influence postoperative blood loss in TKA (8).

Primary TKA in our department is performed according to a strict standardized protocol, which has been respected for all the patients included in this study. All were operated by a single surgeon (ET), following the same minimally invasive technique (medial subvastus arthrotomy) with a Postero-Stabilized cemented implant (Vanguard PS or Persona PS, Zimmer Biomet). An intramedullary femoral resection guide and an extra-medullary guide for the tibia were used. The patella is routinely resurfaced unless the antero-posterior thickness is less than $12 \mathrm{~mm}$.

Pre-operative intravenous injection of $1 \mathrm{~g}$ tranexamic acid and peri-operative local injection of Ropivacaine + Adrenaline are performed routinely. In the first group, the tourniquet was not used or, in case of a venous-tourniquet, was deflated within few minutes of the surgical approach and not inflated again until the end of surgery. In the second group, after exsanguination with an Esmarch bandage, a tourniquet was used (mean pressure 270 $\mathrm{mm} \mathrm{Hg}$ ) during the procedure and released before the closure.

Patients are allowed to flex the operated knee at D0 and walking reeducation with the physiotherapist starts on D1.

Biological controls are performed at D2 and D4 after surgery and patients left routinely the hospital at D4 after surgery. Follow-up consultations are at 3,6 and 12 weeks after surgery.

TBL in TKA can be divided into visible and nonvisible blood loss. Visible blood loss is the sum of intra-operative bleeding and the content of surgical drains, if used. Non-visible blood loss, also known as HBL, represents delayed post-operative loss. It is called "hidden" because it is not possible to quantify it directly, it can only be calculated using the formula : $\mathrm{HBL}=\mathrm{TBL}-$ visible blood loss. Since we never use drains, the above-mentioned formula can be resumed as: $\mathrm{HBL}=\mathrm{TBL}$ - intra-operative blood loss. Nevertheless, measuring intraoperative blood loss is a delicate task because it requires weighing the sponges and noting the volume of aspirated blood. Since the volume of blood aspirated also includes all the other fluids used during the surgical intervention (anesthetic infiltration, pulsed lavage fluid), it generates a large margin of error in the calculation of HBL. This is why the option to quantify peri-operative blood loss as the drop in $\mathrm{Hb}$ level between the pre-operative value and the values at Day 2 or Day 4 was chosen in the present study. The first drop in $\mathrm{Hb}$, measured at day 2 is considered early blood loss, containing mainly the visible blood loss. The second drop in $\mathrm{Hb}$, between Day 2 and Day 4 is considered as $\operatorname{HBL}(6,8)$.

Thus, based on the Gross formula (9), TBL was calculated using the Hb-balance equation, which is the most reliable way to calculate TBL (10) : Total volume of blood loss $(\mathrm{mL})=$ Body Volume $\mathrm{x}([\mathrm{Hb}$ initial $-\mathrm{Hb}$ post-operative $] / \mathrm{Hb}$ initial).

(Body Volume $=\mathrm{k}_{1} \times$ Height $^{3}+\mathrm{k}_{2} \times$ Weight $+\mathrm{k}_{3}$.

For males, $\mathrm{k}_{1}=0,3669, \mathrm{k}_{2}=0,03219, \mathrm{k}_{3}=0,6041$, while for females, $\mathrm{k}_{1}=0,3561, \mathrm{k}_{2}=0,03308, \mathrm{k}_{3}=$ 0,1833) (9).

Preoperative $\mathrm{Hb}$ value was checked 2 weeks before surgery during preoperative anesthesiology consultation.

$\mathrm{Hb}$ values were analyzed at D2, D4 and D42 after surgery, and D2 and D4 values were compared in order to detect any delayed HBL. Concerning TBL, the $\mathrm{Hb}$-balance equation was applied at D4 after surgery, usual day of return home which is included in the nadir HTC range of day (11).

Transfusion trigger and volume of transfusion are taken into account in the TBL calculation. The transfusion threshold was set at a restrictive level of $8 \mathrm{~g} / \mathrm{dL}$ and all patients were followed by an internist during the hospitalization who decided on the indication for transfusion depending on the individual patient's comorbidities and clinical symptoms.

Functional outcome was evaluated 3 months after surgery by 2 questionnaires filled by patients themselves : the Forgotten Joint score (FJS-12) and the Knee injury and Osteoarthritis Outcome Score (KOOS).

Results are presented as numbers, means and standard deviations (SD). Statistical analysis was performed using Student T-test, with a significance set at $\mathrm{P}$ less than 0.05 . 


\begin{tabular}{|c|c|c|c|c|c|}
\hline \multicolumn{6}{|c|}{ No tourniquet } \\
\hline \multirow{2}{*}{\multicolumn{2}{|c|}{$\mathrm{Hb}(\mathrm{g} / \mathrm{dll})$}} & \multirow[b]{2}{*}{ Pre operative } & \multicolumn{3}{|c|}{ Post operative } \\
\hline & & & Day 2 & Day 4 & Day 42 \\
\hline \multirow{2}{*}{$\begin{array}{l}\text { Total } \\
(n=54)\end{array}$} & mean $(S D)$ & $13,3(1,5)$ & $10,8(1,4)$ & $10,3(1,3)$ & $12,2(1,4)$ \\
\hline & median & 13,4 & 11,0 & 10,3 & 12,0 \\
\hline \multirow{2}{*}{$\begin{array}{c}\text { Men } \\
(n=18)\end{array}$} & mean $(S D)$ & $13,8(1,1)$ & $11,3(1,0)$ & $10,7(0,9)$ & $12,6(1,4)$ \\
\hline & median & 13,7 & 11,2 & 11,0 & 11,9 \\
\hline \multirow{2}{*}{$\begin{array}{l}\text { Women } \\
(n=36)\end{array}$} & mean (SD) & $13,0(1,6)$ & $10,6(1,5)$ & $10,1(1,4)$ & $12,9(1,3)$ \\
\hline & median & 13,1 & 10,9 & 9,9 & 12,1 \\
\hline
\end{tabular}

\begin{tabular}{|c|r|c|ccc|}
\hline \multicolumn{2}{|c|}{} & \multicolumn{4}{|c|}{ Tourniquet } \\
& \multicolumn{4}{|c|}{ Post operative } \\
\hline $\begin{array}{c}|c| \\
\text { Total }\end{array}$ & mean (SD) & $13,7(1,4)$ & $11,9(1,4)$ & $11,5(1,5)$ & $13,2(1,4)$ \\
$(\mathrm{n}=110)$ & median & 13,8 & 11,9 & 11,4 & 13,1 \\
\hline Men & mean (SD) & $14,5(1,4)$ & $12,4(1,3)$ & $12,1(1,4)$ & $13,7(1,4)$ \\
$(\mathrm{n}=64)$ & median & 14,5 & 12,3 & 12,1 & 13,8 \\
\hline Women & mean (SD) & $13,2(1,0)$ & $11,2(1,1)$ & $10,7(1,1)$ & $12,5(0,9)$ \\
$(\mathrm{n}=46)$ & median & 13,3 & 11,1 & 10,8 & 12,5 \\
\hline
\end{tabular}
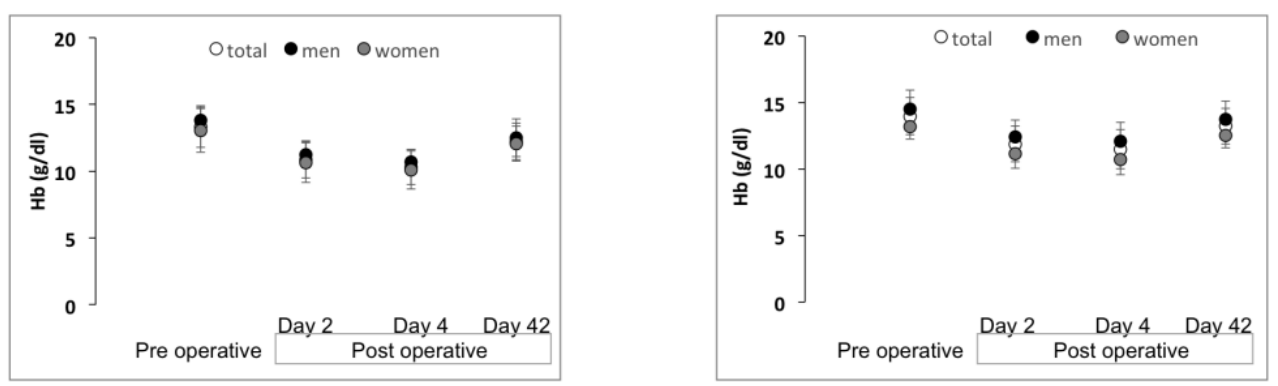

Figure 1. - Hb concentrations as means (standard deviation) and medians for both groups (with and without tourniquet). Means and standard deviation are represented in graphs below the tables.

\begin{tabular}{|c|c|c|c|c|c|c|}
\hline \multirow{2}{*}{$\begin{array}{c}\text { Total blood } \\
\text { loss (ml) }\end{array}$} & \multicolumn{2}{|c|}{ Total } & \multicolumn{2}{c|}{ Men } & \multicolumn{2}{c|}{ Women } \\
\cline { 2 - 7 } & No tourniquet & Tourniquet & No tourniquet & Tourniquet & No tourniquet & Tourniquet \\
\hline mean (SD) & $1073(366)$ & $890(358)$ & $1200(399)$ & $943(396)$ & $1010(336)$ & $817(284)$ \\
median & 1081 & 914 & 1147 & 939 & 1017 & 806 \\
\hline p-value & \multicolumn{2}{|c|}{0,003} & \multicolumn{2}{|c|}{0,019} & \multicolumn{2}{c}{0,008} \\
\hline
\end{tabular}

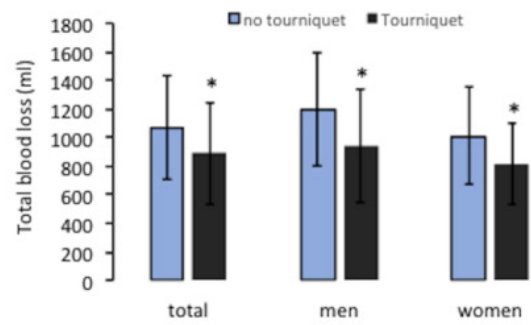

Figure 2. - Means, standard deviations and medians of total blood loss for men, women and both genders compared between the two groups. * : statistical difference between no tourniquet and tourniquet group, p-value $<0.05$.

\section{RESULTS}

Perioperative blood loss is represented as the difference between $\mathrm{Hb}$ preoperative value and $\mathrm{Hb}$ measured on Day 2. HBL is represented as the difference between $\mathrm{Hb}$ values at Day 2 and Day 4. Hb-balance formula applied to $\mathrm{D} 4 \mathrm{Hb}$ value represents the TBL. The obtained values were then compared in both groups.
Concerning HBL, differences between both groups were not significant $(0,5 \mathrm{vs} 0,4 \mathrm{~g} / \mathrm{dL}$ ) (see Figure 1)

The difference of TBL between the two groups is statistically significant (1073 vs $890 \mathrm{ml}$ ) (See Figure 2)

2 patients received allogenic red cells transfusion in the no-tourniquet group (4\%) during hospitalization. None of the patients in the tourniquet group was transfused $(0 \%)$. 
Table II. - Summary of functional outcomes scores for men, women and both genders in our series.

\begin{tabular}{|c|c|c|c|c|c|c|c|}
\hline \multicolumn{8}{|c|}{ No tourniquet } \\
\hline \multirow{2}{*}{\multicolumn{2}{|c|}{$\begin{array}{l}\text { Normalized score } \\
\text { from } 0 \text { to } 100\end{array}$}} & \multicolumn{2}{|c|}{ Total } & \multicolumn{2}{|c|}{ Men } & \multicolumn{2}{|c|}{ Women } \\
\hline & & mean (SD) & median & mean $(S D)$ & median & mean (SD) & median \\
\hline \multicolumn{2}{|c|}{ Forgotten joint } & $50(27)$ & 52 & $38(20)$ & 40 & $58(29)$ & 67 \\
\hline \multirow{5}{*}{ Koos } & Symptoms & $67(19)$ & 68 & $58(22)$ & 57 & $73(14)$ & 75 \\
\hline & Pain & $75(20)$ & 82 & $72(20)$ & 81 & $78(20)$ & 86 \\
\hline & $A D L$ & $72(19)$ & 74 & 65 (18) & 72 & 77 (19) & 75 \\
\hline & Sport/rec & $43(27)$ & 30 & $55(32)$ & 55 & 30 (14) & 25 \\
\hline & QOL & $55(25)$ & 56 & $52(30)$ & 50 & $56(22)$ & 59 \\
\hline \multicolumn{8}{|c|}{ Tourniquet } \\
\hline \multirow{2}{*}{\multicolumn{2}{|c|}{$\begin{array}{l}\text { Normalized score } \\
\text { from } 0 \text { to } 100\end{array}$}} & \multicolumn{2}{|c|}{ Total } & \multicolumn{2}{|c|}{ Men } & \multicolumn{2}{|c|}{ Women } \\
\hline & & mean (SD) & median & mean (SD) & median & mean $(S D)$ & median \\
\hline \multirow{6}{*}{ kOOS } & gotten joint & $60(24)$ & 63 & $61(21)$ & 63 & $57(27)$ & 60 \\
\hline & Symptoms & $73(16)$ & 75 & $73(12)$ & 72 & $72(20)$ & 79 \\
\hline & Pain & $77(18)$ & 83 & $78(16)$ & 83 & $77(20)$ & 83 \\
\hline & ADL & $78(17)$ & 81 & 79 (14) & 79 & $78(21)$ & 87 \\
\hline & Sport/rec & $55(25)$ & 60 & $48(19)$ & 45 & $69(29)$ & 85 \\
\hline & QOL & $68(25)$ & 75 & $65(24)$ & 69 & $72(26)$ & 81 \\
\hline
\end{tabular}
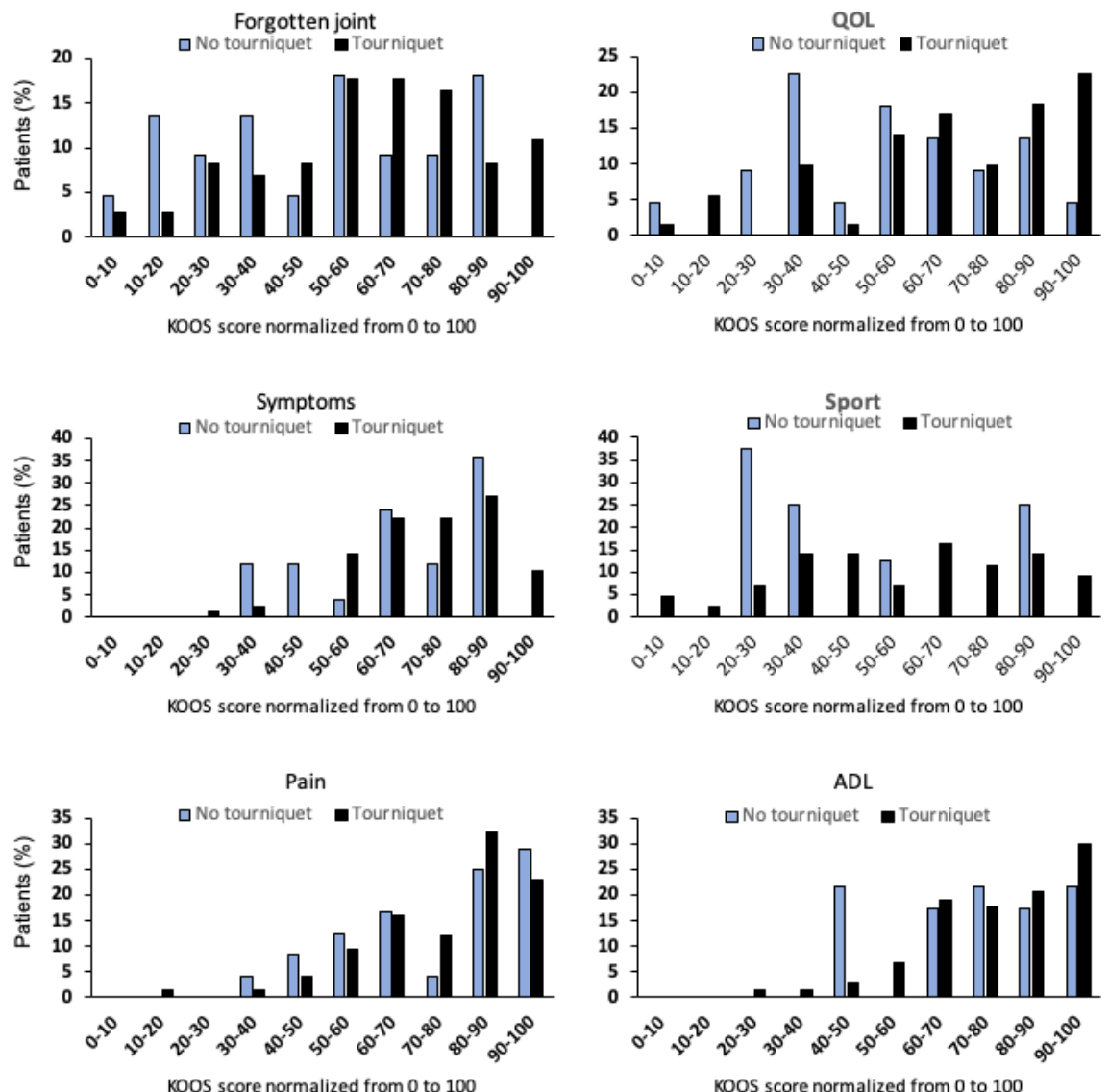

Figure 3. - Functional scores are represented on graphs showing the percentage of patients for each score interval in both groups. 
Patients were questioned about functional outcomes 3 months after surgery. In order to evaluate these outcomes, we used the Forgotten Joint score (FJS-12) and the Knee injury and Osteoarthritis Outcome Score (KOOS), both validated after primary TKA $(12,13)$.

The FJS-12 assesses awareness of the knee joint during various activities of daily living (ADL) following joint replacement. This test is a unidimensional interval-level scale composed by 12 items which evaluate the ability to forget the joint after arthroplasty.

The Knee Injury and Osteoarthritis Outcome Score (KOOS) is a questionnaire designed to assess short and long-term patient-relevant outcomes following knee injury. The KOOS is self-administered and assesses five outcomes : pain, symptoms, activities of daily living, sport and recreation function, and knee-related quality of life. The KOOS can be used to evaluate the course of treatment outcome, such as TKA.

KOOS values have been normalized to meet the scale usually used in orthopedics according to the KOOS Manual Score calculation (2012) : 100 indicates no problems and 0 extreme problems. FJS-12 results were normalized in the same fashion.

All FJS and KOOS scores are given into Table II and compared between the two groups into Figure 3.

\section{DISCUSSION}

Our data show an absence of delayed hidden blood loss and a lower total blood loss in the tourniquet group.

The main limitation of our study is the selection of the control group. Indeed, part of these patients have been operated without tourniquet because of specific illnesses who made tourniquet-use at risk. These comorbidities induce a bias in the results of functional outcome that is potentially reduced by pre-operative poorer conditions, especially in the FJS score and KOOS QOL. Concerning the blood loss, one could argue that after a venous tourniquet, soft tissues are filled with venous blood and are likely to increase the visible blood loss during the surgery.
Finally, as patients usually left the hospital at $\mathrm{D} 4$, no subsequent $\mathrm{Hb}$ values is known. The value measured on Day 4 is usually the lowest during the hospital stay and it is used to calculate the total blood loss. But Prasad et al. showed that the nadir HTC value appears between D4 and D7 after surgery, so our TBL is possibly underestimated (11).

The choice to routinely use tourniquet must be balanced between theoretical advantages and side effects. The literature is very controversial about this topic and does not allow to develop clear guidelines $(1,14)$. Indeed, many tourniquetrelated complications have been described. Muscle compression under tourniquet leads to muscle ischemia, which could cause quadriceps weakness, reperfusion injury and postoperative thigh pain. Compression of neuro-vascular structures carry a risk for vascular injuries, deep vein thrombosis and nerve-related injuries. These side effects all lead to a slower recovery and important co-morbidity (5).

Over time, tourniquet use has evolved along with knee surgery techniques, and certainly that of TKA. The modernization of instrumentation has reduced the procedure time and therefore the time of tourniquet use.

The shape of pneumatic tourniquet has also been investigated and we know that a wide cuff, like the one used in our protocol, is more effective in the vessel occlusion stage and can be less painful if the inflate-pressure is limited (15). The inflate tourniquet-pressure is directly correlated to the rate of complications (5). It is therefore logical to suppose that decreasing the tourniquet-inflate pressure would decrease these complications, however very few papers have demonstrated this $(16,17)$. Interestingly, lower tourniquet-inflate pressures used nowadays are certainly one of the reasons why recent studies show very different results from previous ones concerning TKA complications. For example, Stringer et al. in 1989 reported a dramatic rate of $56 \%$ deep vein thrombosis (DVT) after TKA, but the tourniquet-pressure was $500 \mathrm{~mm} \mathrm{Hg}$ (18). In contrast, a meta-analysis carried out in 2019 , revealed that tourniquet-use did not significantly increase the incidence of DVT (19).

In our series, a conventional tourniquet that keeps an initial setting pressure during surgery was used. 
Values of $280 \mathrm{~mm} \mathrm{Hg}$ for men and $260 \mathrm{~mm} \mathrm{Hg}$ for women were chosen giving us a mean tourniquet pressure of $270 \mathrm{~mm} \mathrm{Hg}$ in our study. Most of the time, this technique allows the tourniquet to be used without failure. Routinely, if a venous-tourniquet happens during the surgery, the tourniquet deflation is preferred and the procedure continues without tourniquet.

Goel et al. recently showed lower rates of total blood loss in tourniquet groups compared to notourniquet groups (20). Their tourniquet-cohort results are similar to ours, but differ from older studies. The modern multimodal approach to prevent blood loss in TKA could explain these differences. In the present study, besides the lowpressure tourniquet-use, our TBL reduction is due to several measures respected in our TKA protocol. This protocol has an anesthetic side with improved analgesia and peri-operative blood pressure regulation. Furthermore, a pre-operative intravenous injection of $1 \mathrm{~g}$ tranexamic acid is given. This antifibrinolytic agent has been studied for years and is a reference in peri-operative blood loss prevention $(21-24)$. On the surgical side, each step is followed in an overall desire to avoid bleeding. First, even if the role of minimally invasive TKA has not been clearly determined about blood loss (25), a minimal medial subvastus arthrotomy is used, allowing quadricipital tendon sparing. The femoral entry point of the intramedullary guide is plugged with a bone autograft before the definitive implants cementation (26).

Shen et al. showed that deflating the tourniquet before closure reduces the total blood loss allowing final hemostasis (27). It is obviously a way to limit tourniquet time, but also an indirect way to reduce limb girth by blood diffusion into soft tissues under the compressive bandage (28). Li et al. (2) attributed $50 \%$ of TBL to HBL in their series where they did not release the tourniquet before closure. This final check is always performed during the last minutes of cementation in our protocol and can partially explain our very low HBL rate.

In the tourniquet group, our results are very good concerning pain and function in daily living, and good concerning symptoms and quality of life. It means good functional outcome at 3 months can be obtained after surgery. Poor KOOS-sport results are explained by the mean cohort age and its major absence of pre-operative sport in daily life. In addition, most sports are not recommended at this time after the operation.

Jahwar et al. have recently shown in a prospective study no difference in functional outcome using or not a tourniquet for primary TKA (16) even with a high pressure of $360 \mathrm{~mm} \mathrm{Hg}$. Recovery after TKA is certainly more influenced by all perioperative measures than by the use or not of the tourniquet.

\section{CONCLUSION}

This study shows that tourniquet-use, at low pressure and integrated in a multimodal standardized protocol, reduces TBL and does not lead to HBL in the post-operative period after primary TKA.

\section{Acknowledgments}

I would like to thank Edith Renguet for her careful proofreading of my english and her expertise in scientific publications.

\section{REFERENCES}

1. Jiang, F.Z., et al., Use of a tourniquet in total knee arthroplasty: a systematic review and meta-analysis of randomized controlled trials. J Orthop Sci, 2015. 20(1) : 110-23.

2. Li, B., et al., The effect of tourniquet use on hidden blood loss in total knee arthroplasty. Int Orthop, 2009. 33(5) : 1263-8.

3. Sehat, K.R., R.L. Evans, J.H. Newman. Hidden blood loss following hip and knee arthroplasty. Correct management of blood loss should take hidden loss into account. J Bone Joint Surg Br, 2004. 86(4) : 561-5.

4. Du, Z., et al., [Effect of tourniquet on perioperative blood loss and short-term effectiveness in total knee arthroplasty]. Zhongguo Xiu Fu Chong Jian Wai Ke Za Zhi, 2013. 27(11) : 1318-23.

5. Smith, T.O., C.B. Hing. Is a tourniquet beneficial in total knee replacement surgery? A meta-analysis and systematic review. Knee, 2010. 17(2) : 141-7.

6. Schwab, P.E., et al., Lower blood loss after unicompartmental than total knee arthroplasty. Knee Surg Sports Traumatol Arthrosc, 2015. 23(12) : 3494-500.

7. Spahn, D.R. Anemia and patient blood management in hip and knee surgery : a systematic review of the literature. Anesthesiology, 2010. 113(2) : 482-95. 
8. Schwab, P.E., et al., Aspirin mono-therapy continuation does not result in more bleeding after knee arthroplasty. Knee Surg Sports Traumatol Arthrosc, 2017. 25(8) : 25862593.

9. Gross, J.B. Estimating allowable blood loss: corrected for dilution. Anesthesiology, 1983. 58(3) : 277-80.

10. Gao, F.Q., et al., Four Methods for Calculating Blood-loss after Total Knee Arthroplasty. Chin Med J (Engl), 2015. 128(21) : 2856-60.

11. Prasad, N., V. Padmanabhan, A. Mullaji. Blood loss in total knee arthroplasty: an analysis of risk factors. Int Orthop, 2007. 31(1) : 39-44.

12. Thomsen, M.G., et al., Good validity and reliability of the forgotten joint score in evaluating the outcome of total knee arthroplasty. Acta Orthop, 2016. 87(3) : 280-5.

13. Roos, E.M., L.S. Lohmander. The Knee injury and Osteoarthritis Outcome Score (KOOS): from joint injury to osteoarthritis. Health Qual Life Outcomes, 2003. 1 : 64.

14. Li, X., et al., The effect of tourniquet use in total knee arthroplasty: grading the evidence through an updated metaanalysis of randomized, controlled trials. Eur J Orthop Surg Traumatol, 2014. 24(6) : 973-86.

15. Estebe, J.P., et al., Tourniquet pain in a volunteer study: effect of changes in cuff width and pressure. Anaesthesia, 2000. 55(1) : 21-6.

16. Jawhar, A., et al., No effect of tourniquet in primary total knee arthroplasty on muscle strength, functional outcome, patient satisfaction and health status: a randomized clinical trial. Knee Surg Sports Traumatol Arthrosc, 2020. 28(4) : 1045-1054.

17. Olivecrona, C., et al., Lower tourniquet cuff pressure reduces postoperative wound complications after total knee arthroplasty: a randomized controlled study of 164 patients. J Bone Joint Surg Am, 2012. 94(24) : 2216-21.

18. Stringer, M.D., et al., Deep vein thrombosis after elective knee surgery. An incidence study in 312 patients. $J$ Bone Joint Surg Br, 1989. 71(3) : 492-7.
19. Cai, D.F., et al., The effects of tourniquet use on blood loss in primary total knee arthroplasty for patients with osteoarthritis: a meta-analysis. J Orthop Surg Res, 2019. $14(1): 348$.

20. Goel, R., et al., Tourniquet Use Does Not Affect Functional Outcomes or Pain After Total Knee Arthroplasty : A Prospective, Double-Blinded, Randomized Controlled Trial. $J$ Bone Joint Surg Am, 2019. 101(20) : 1821-1828.

21. White, C.C.t., J.K. Eichinger, R.J. Friedman. Minimizing Blood Loss and Transfusions in Total Knee Arthroplasty. $J$ Knee Surg, 2018. 31(7) : 594-599.

22. Ker, K., et al., Effect of tranexamic acid on surgical bleeding: systematic review and cumulative meta-analysis. BMJ, 2012. 344 : e3054.

23. Moskal, J.T., R.N. Harris, S.G. Capps. Transfusion cost savings with tranexamic acid in primary total knee arthroplasty from 2009 to 2012. J Arthroplasty, 2015. $30(3): 365-8$.

24. Alshryda, S., et al., Tranexamic acid in total knee replacement: a systematic review and meta-analysis. $J$ Bone Joint Surg Br, 2011. 93(12) : 1577-85.

25. Khanna, A., et al., Minimally invasive total knee arthroplasty: a systematic review. Orthop Clin North Am, 2009. 40(4) : 479-89, viii.

26. Kumar, N., et al., Plugging the intramedullary canal of the femur in total knee arthroplasty: reduction in postoperative blood loss. J Arthroplasty, 2000. 15(7) : 947-9.

27. Shen, H.L., et al., Analysis on hidden blood loss of total knee arthroplasty in treating knee osteoarthritis. Chin Med $J$ (Engl), 2011. 124(11) : 1653-6.

28. Zan, P.F., et al., Releasing of tourniquet before wound closure or not in total knee arthroplasty: a meta-analysis of randomized controlled trials. J Arthroplasty, 2015. 30(1) : 31-7. 